\title{
Planar Bipedal Robot with Impulsive Foot Action
}

\author{
Jun Ho Choi and J. W. Grizzle
}

\begin{abstract}
A planar bipedal robot with an impulsive actuator at each foot is considered. The analysis extends previous work on a model with unactuated point feet [1] to include the actuator model of [2]. The impulsive actuator at each foot is active only during the double support phase, which results in the model being identical to the model with unactuated point feet for the single support phase. However, the impulsive foot actuation results in a different model for the double support map. Conditions for the existence of a hybrid zero dynamics for the robot with foot actuation are studied. A feedback design method is proposed that integrates actuation in the single and double support phases. A stability analysis is performed using a Poincaré return map.
\end{abstract}

\section{INTRODUCTION}

In this paper, a planar bipedal robot with impulsive foot action is studied. The impulsive foot actuator is assumed to be active during double support phases. This is an approximation of the toe-off action observed in double support phases of human walking, [3]. Although the impulsive foot action is not necessary for stable walking as shown in [4], [5], and [1], having the impulsive foot action shows the possibility of more efficient walking, [6] and [2].

Grizzle et al., for planar robot models with one degree of underactuation in single support, introduced a feedback method whereby existence and stability of periodic orbits could be determined by a one-dimensional map. A key aspect of the analysis uses the notion of virtual constraints, that is, holonomic constraints that are asymptotically imposed on the robot's motion via feedback control. Westervelt $e t$ al. introduced the notion of the hybrid zero dynamics, which transformed the method of virtual constraints into a practical design method, capable of treating N-link planar bipedal robots with point feet and one degree of underactuation. Extensive experiments have been reported in [7] and [8].

Control approaches for robots vary with the characteristics of the feet. McGeer analyzed a robot that had arcshaped unactuated feet in [6]. Yi studied a robot that had a passive ankle, specifically, the ankle joints were connected with springs in [9]. In [10], Linde investigated a 2-link robot that had a variable spring and damper at each leg with arcshaped unactuated feet. Many papers in the literature have considered actuated feet with nontrivial length. In [11], [12], [13], the sole of the stance foot remains on the ground during the single-support phase. Since keeping the support sole on the ground limits the motion of the robot, it was suggested to allow the foot to roll with respect to the stance toe [14], [15]. Impulsive foot actuation was implemented in

Jun Ho Choi and J. W. Grizzle are with the Department of Electrical Engineering and Computer Science, University of Michigan, Ann Arbor, Michigan 48109-2122, USA \{junhoc, grizzle\}@umich.edu a passive robot with round feet. A motor to extend each ankle was located at the ankles. The toe-off power was applied right after the heel strike [16].

A model with impulsive point feet has been studied by Kuo, who investigated the energetics of powered locomotion using a robot with impulsive point feet [2]. The robot was planar and consisted of 2 rigid massless links. An impulsive foot actuator was attached at the end of the stance leg. The impulsive toe-off actuation was applied just before heel strike. Using the model, it was shown that applying toe-off force is more energy efficient than using hip torque alone.

The model studied in this paper is extended from [1] by adding at each foot the impulsive actuator of [2]. The impulsive foot actuator is assumed to be active during the double support phase. This results in the identical model of [1] during the single support phase. However, due to the impulsive foot actuator during the double support phase, the double support map becomes a function of not only the states before the double support phase but also the foot force during the double support phase. The notion of the hybrid zero dynamics is extended to accommodate the presence of the foot force. A class of foot force control laws is introduced to create the invariance of the zero dynamics. The Poincaré map is also used to analyze the stability of the robot's motion.

\section{ROBOT MODEL WITH IMPULSIVE FEET}

The model considered in this paper is extended from the model with unactuated point feet [1] by adding an impulsive actuator at each foot. The robot is assumed to be planar, bipedal, with $\mathrm{N}$ rigid links. Walking is assumed to consist of two phases, which are single support and double support. It is assumed that the impulsive actuator at the stance foot is activated only in the double support phase. Therefore, during the single support phase, the model is identical to a robot with unactuated point feet. The detailed assumptions for the single support phase are based on [1] and are listed in Appendix A for the convenience of the readers.

During the single support phase, the robot is still underactuated since the impulsive foot actuation occurs during the double support phase. Therefore, the model for the single support phase is identical to the one with unactuated point feet, which is given as

$$
D(q) \ddot{q}+C(q, \dot{q}) \dot{q}+G(q)=B u,
$$

where $q=\left(q_{1}, \cdots, q_{N}\right)^{\prime} \in \mathcal{Q}$, a simply connected subset of $[0,2 \pi)^{N}$, are the configuration variables, $\dot{q}$ are the velocities, and $u=\left(u_{1}, \cdots, u_{N-1}\right)^{\prime}$ are the inputs applied at the joints. Let $x=\left(q^{\prime}, \dot{q}^{\prime}\right)^{\prime} \in T \mathcal{Q}$ then the dynamic equation in state-space form is given by $\dot{x}=f(x)+g(x) u$. 


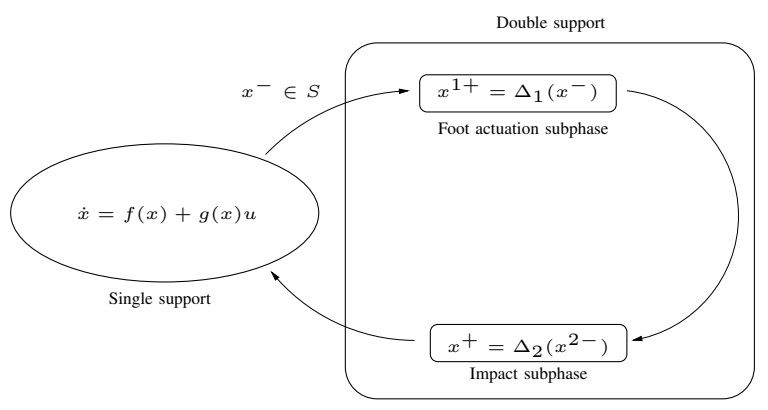

Fig. 1. Diagram for the system, which combines the point foot model [1] with the impulsive foot action [2].

Property 1: Under hypothesis RH5 in Appendix A, that is the coordinates of the robot consist of $N-1$ relative angles, $q_{1}, \cdots, q_{N-1}$, and one absolute angle $q_{N}$, the angular momentum of the robot about the stance foot during the single support phase is $d_{N}(q) \dot{q}$, where $d_{N}(q)$ is the last row of $D$ in (1).

Proof: See Appendix B.

During the double support phase, the foot actuation subphase and the impact subphase occur successively with an infinitesimally small time interval. The foot actuation subphase is when the actuator at the stance foot is active. It applies an impulsive force to the robot in the direction of the stance tibia, which causes a discontinuous change in the velocity states. The impact subphase is when the swing leg touches the ground after the foot actuation. The impact also causes an impulsive reaction force to the robot, which results in another discontinuous change in the velocity states. In both cases the position states remain continuous. The overall double support phase becomes an algebraic mapping that maps the states just before the foot actuation to the states right after the impact. The double support map depends not only on the states before the foot actuation but also on the applied foot force.

Based on Kuo, [2], the necessary hypotheses for the impulsive foot action are :

IFH1) The double support phase lasts for an infinitesimally small period of time;

IFH2) The double support phase has two sequential subphases, which are the foot actuation subphase and the impact subphase;

IFH3) The foot actuation subphase occurs just before the impact subphase, with the time interval between the two subphases being infinitesimally small;

IFH4) There is an impulsive foot actuator at the end of each tibia, which is activated only during the foot actuation subphase;

IFH5) Impulsive foot force causes discontinuous changes in the velocity states but the position states remain continuous;

IFH6) The foot force is applied along the stance tibia, that is, the direction of the force is the same as the stance tibia;
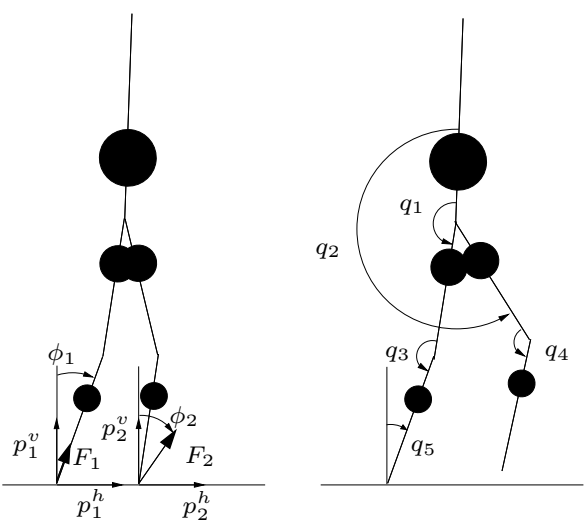

Fig. 2. 5-link robot with impulsive feet during the double support phase and the single support phase. $F_{1}$ and $F_{2}$ represent the impulsive foot force and the impulsive reaction force due to impacts, respectively.

IFH7) The applied foot force is unilateral, in other words, it cannot pull the robot down to the ground.

To describe the double support phase, it is necessary to use an N+2 DOF model (e.g. N DOF for the joints and 2 DOF for the position of the stance end). Adding Cartesian coordinates, $\left(p_{1}^{h}, p_{1}^{v}\right)$, to the end of the stance leg gives $q_{e}=$ $\left(q^{\prime}, p_{1}^{h}, p_{1}^{v}\right)^{\prime}$ and $\dot{q}_{e}=\left(\dot{q}^{\prime}, \dot{p}_{1}^{h}, \dot{p}_{1}^{v}\right)^{\prime}$, see Fig. 2. Let $\Upsilon_{1}\left(q_{e}\right)$ and $\Upsilon_{2}\left(q_{e}\right)$ denote the Cartesian coordinates of the stance foot and the swing foot. Let $\phi_{1}$ and $\phi_{2}$ be the angles of the foot actuation force and the impulsive ground reaction force, computed with respect to the vertical. Using the method of Lagrange, one can obtain the dynamics for the double support phase as follows:

$D_{e}\left(q_{e}\right) \ddot{q}_{e}+C_{e}\left(q_{e}, \dot{q}_{e}\right) \dot{q}_{e}+G_{e}\left(q_{e}\right)=B_{e} u+E_{e}^{1} \delta F_{1}+E_{e}^{2} \delta F_{2}$,

where $E_{e}^{1}=\left(\frac{\partial \Upsilon_{1}\left(q_{e}\right)}{\partial q_{e}}\right)^{T}, E_{e}^{2}=\left(\frac{\partial \Upsilon_{2}\left(q_{e}\right)}{\partial q_{e}}\right)^{T}$, and $\delta F_{1}$ and $\delta F_{2}$ denote the impulsive foot force and the impulsive ground reaction force, respectively.

During the foot actuation subphase, $F_{1}=\mathcal{T} \delta F_{f}=$ $\left[\begin{array}{c}\sin \phi_{1} \\ \cos \phi_{1}\end{array}\right] \delta F_{f}$ and $\delta F_{2}=0$. Let $q_{e}^{1}$ be the state vector for the foot actuation subphase. Then, following the procedure in [5], (2) becomes

$$
D_{e}\left(\dot{q}_{e}^{1+}-\dot{q}_{e}^{1-}\right)=E_{e} \mathcal{T} F_{f},
$$

where $F_{f}=\int \delta F_{f} d t, \dot{q}_{e}^{1+}$ is the velocity just after the foot actuation, and $\dot{q}_{e}^{1-}$ is the velocity just before the foot actuation. Since the stance foot acts as a pivot before the double support phase, $\dot{q}_{e}^{1-}=\left(\dot{q}^{-}, 0,0\right)^{\prime}$.

Property 2: Under hypotheses RH5 and GH2 in Appendix $\mathrm{A}, d_{N}\left(q^{-}\right) \dot{q}^{-}=d_{e, N}\left(q_{e}^{-}\right) \dot{q}_{e}^{-}$, where $d_{e, N}\left(q_{e}\right)$ is the $N$ th row of $D_{e}$ in (2).

Proof: Let $L$ and $L_{e}$ denote the Lagrangians for the single support phase and double support phase, respectively. Define $L=K-V$ and $L_{e}=K_{e}-V_{e}$, where $K$ and $K_{e}$ are the kinetic energies and $V$ and $V_{e}$ are the potential energies. By the hypothesis $\mathrm{GH} 2$, $L=\left.L_{e}\right|_{\left(p_{1}^{h}, p_{1}^{v}, \dot{p}_{1}^{h}, \dot{p}_{1}^{v}\right)}=(c, 0,0,0)$, where $c$ is constant. 
Since $d_{N}(q) \dot{q}=\partial L / \partial \dot{q}_{N}, d_{N}\left(q^{-}\right) \dot{q}^{-}=\partial L / \partial \dot{q}_{N}=$ $\partial L_{e} /\left.\partial \dot{q}_{N}\right|_{\left(p_{1}^{h}, p_{1}^{v}, \dot{p}_{1}^{h}, \dot{p}_{1}^{v}\right)=(c, 0,0,0)}=d_{e, N}\left(q_{e}^{-}\right) \dot{q}_{e}^{-}$

Property 3: Assume the hypothesis RH5 in Appendix $\mathrm{A}$, then in the coordinates $q_{e}^{1}=\left(q_{1}, \cdots, q_{N}, p_{1}^{h}, p_{1}^{v}\right)$, $d_{e, N}\left(q_{e}^{1-}\right) \dot{q}_{e}^{1-}=d_{e, N}\left(q_{e}^{1+}\right) \dot{q}_{e}^{1+}$, where $q_{e}^{1-}, \dot{q}_{e}^{1-}$ are the states before the foot actuation and $q_{e}^{1+}, \dot{q}_{e}^{1+}$ are the states after the foot actuation.

Proof: In the given coordinate, the Cartesian coordinate of the stance foot is given by $\Upsilon_{1}\left(q_{e}\right)=\left(p_{1}^{h}, p_{1}^{v}\right)$. Therefore, $E_{N}=\left(\partial \Upsilon_{1} / \partial q_{N}\right)^{T}=0$. Thus, $d_{e, N}\left(q_{e}^{1+}\right) \dot{q}_{e}^{1+}-$ $d_{e, N}\left(q_{e}^{1-}\right) \dot{q}_{e}^{1-}=0$.

Properties 2 and 3 imply the angular momentum about the stance foot is conserved even with foot actuation at the stance foot.

Property 4: Let $\vec{P}^{+}, \vec{P}_{c}^{-}$be the linear momentum of the robot after and before foot actuation. Then $\vec{P}^{+}-\vec{P}_{c}^{-}=\mathcal{T} F_{f}$

Proof: See Appendix C

Since $D_{e}$ in (3) is positive definite, it is always possible to calculate the velocity states after the foot actuation. The velocity states after the foot actuation are given as

$$
\dot{q}_{e}^{1+}=\dot{q}_{e}^{1-}+\left(D_{e}\right)^{-1} E_{e}^{1} \mathcal{T} F_{f} .
$$

Right after the foot force is applied to the stance leg, the swing leg makes contact with the ground, which is the impact subphase. During the impact subphase, $\delta F_{1}=0$ and $\delta F_{2}=\left[\begin{array}{l}\delta F_{2}^{T} \\ \delta F_{2}^{N}\end{array}\right]$, where $\delta F_{2}^{T}$ and $\delta F_{2}^{N}$ denote the tangential and normal components of the impulsive reaction force. Following the procedure in [5] again, (2) becomes

$$
D_{e}\left(\dot{q}_{e}^{2+}-\dot{q}_{e}^{2-}\right)=E_{e}^{2} F_{2},
$$

where $F_{2}=\int \delta F_{2} d t, \dot{q}_{e}^{2+}$ is the velocity just after the impact, and $\dot{q}_{e}^{2-}$ is the velocity just before the impact.

Property 5: Under hypotheses RH5 and GH2 in Appendix A, $d_{N}\left(q^{2+}\right) \dot{q}^{2+}=d_{e, N}\left(q_{e}^{2-}\right) \dot{q}_{e}^{2-}$.

Proof: The proof is analogous to the proof of property 2.

Property 6: Assume the hypothesis RH5 in Appendix A. Then in the coordinate $q_{e}^{2}=\left(q_{1}, \cdots, q_{N}, p_{2}^{h}, p_{2}^{v}\right)$, $d_{e, N}\left(q_{e}^{2-}\right) \dot{q}_{e}^{2-}=d_{e, N}\left(q_{e}^{2+}\right) \dot{q}_{e}^{2+}$, where $q_{e}^{2-}, \dot{q}_{e}^{2-}$ are the states before impact and $q_{e}^{2+}, \dot{q}_{e}^{2+}$ are the states after impact. 3.

Proof: The proof is analogous to the proof of property

Properties 5 and 6 imply the angular momentum about the swing leg end is conserved during the impact of the swing leg end.

By the assumption that the swing leg does not slip after the impact,

$$
\frac{\partial \Upsilon_{2}}{\partial q_{e}} \dot{q}_{e}^{2+}=\left(E_{e}^{2}\right)^{T} \dot{q}_{e}^{2+}=0
$$

Augmenting (5) and (6) yields

$$
\left[\begin{array}{c}
\dot{q}^{2+} \\
F_{2}
\end{array}\right]=\left[\begin{array}{cc}
D_{e} & -E_{e}^{2} \\
\left(E_{e}^{2}\right)^{T} & 0
\end{array}\right]^{-1}\left[\begin{array}{c}
D_{e} \dot{q}_{e}^{2-} \\
0
\end{array}\right] .
$$

By assumption IFH3, there is an infinitesimally small time interval between the foot actuation and the impact, which implies that $q_{e}^{1+}=q_{e}^{2-}$ and $\dot{q}_{e}^{1+}=\dot{q}_{e}^{2-}$. Then the velocity states after the impact can be expressed as a function of the states before the foot actuation and the foot force. After the impact, the states need to be relabeled since the swing leg and the stance leg switch their roles for the next step. The overall mapping from the velocity states just before the foot actuation to the velocity states right after the impact is given as follows:

$$
\begin{aligned}
\dot{q}^{+}= & {\left[\begin{array}{ll}
R & 0
\end{array}\right]\left[\begin{array}{cc}
D_{e} & -E_{e}^{2} \\
\left(E_{e}^{2}\right)^{T} & 0
\end{array}\right]^{-1}\left[\begin{array}{c}
D_{e} \\
0
\end{array}\right]\left[\begin{array}{l}
I \\
0
\end{array}\right] \dot{q}^{-} } \\
& +\left[\begin{array}{ll}
R & 0
\end{array}\right]\left[\begin{array}{cc}
D_{e} & -E_{e}^{2} \\
\left(E_{e}^{2}\right)^{T} & 0
\end{array}\right]^{-1}\left[\begin{array}{c}
E_{e}^{1} \mathcal{T} \\
0
\end{array}\right] F_{f} \\
= & \Delta_{\dot{q} 2}\left(q^{-}\right) \dot{q}^{-}+\Delta_{\dot{q} 1}\left(q^{-}\right) F_{f},
\end{aligned}
$$

where $\dot{q}^{-}, \dot{q}^{+}$are the velocity states just before the foot action and right after the impact, respectively, and $R$ is a relabeling matrix. Since the position states remain continuous during the double support phase, the position states only need to be relabeled for the next step, $q^{+}=\Delta_{q}\left(q^{-}\right)=$ $R q^{-}$. The double support map is given as

$$
\begin{aligned}
x^{+}=\left[\begin{array}{c}
q^{+} \\
\dot{q}^{+}
\end{array}\right] & =\left[\begin{array}{c}
\Delta_{q} q^{-} \\
\Delta_{\dot{q} 2}\left(q^{-}\right) \dot{q}^{-}+\Delta_{\dot{q} 1}\left(q^{-}\right) F_{f}
\end{array}\right] \\
& =\Delta\left(x^{-}, F_{f}\right) .
\end{aligned}
$$

Note that $\Delta_{\dot{q} 2}=\Delta_{\dot{q}}$ in [1] and $\Delta_{\dot{q} 1}\left(q^{-}\right), \Delta_{\dot{q} 2}\left(q^{-}\right)$are functions of the position states only.

Following the method in [17] gives the overall system description. Let $S=\left\{x \in T \mathcal{Q} \mid p_{2}^{h}>0, p_{2}^{v}=0\right\}$ be a set of states satisfying the conditions for the double support phase, where $\left(p_{2}^{h}, p_{2}^{v}\right)$ represents the Cartesian coordinates of the swing foot (see Fig. 2).

$$
\begin{cases}\dot{x}=f(x)+g(x) u, & x \notin S \\ x^{+}=\Delta\left(x^{-}, F_{f}\right), & x^{-} \in S, F_{f} \in \mathbb{R}^{+} \cup\{0\}\end{cases}
$$

Note that the dynamics for the single support phase is identical to the model with unactuated point feet and the double support map becomes identical when the foot force $F_{f}$ is zero.

\section{EXISTENCE OF HYBRID ZERO DYNAMICS}

In this section, the existence of a zero dynamics for the hybrid model (10) is studied. With the foot actuation, the hybrid zero dynamics manifold is determined by not only the choice of the output function but also the foot force applied during the double support phase.

Let $y=h(x)$ be a vector of $N-1$ functions, satisfying HH1-HH5 of [1] (see Appendix A), which essentially means that $h$ depends only on the configuration variables during the single support phase, the associated decoupling matrix, $L_{g} L_{f} h(x)$, is full rank, and $h$ can be completed to a local diffieomorphism on the configuration space. Then there exists a smooth manifold $Z=\{x \in T \mathcal{Q} \mid h(x)=$ $\left.0, L_{f} h(x)=0\right\}$, called the zero dynamics manifold, and $S \cap Z$ is smooth. $S \cap Z$ is one-dimensional if $S \cap Z \neq \emptyset$. In addition to the hypotheses HH1-HH5, if the hypothesis 
RH5 is also satisfied, then the single support phase zero dynamics can be written as

$$
\begin{aligned}
& \dot{\xi}_{1}=\kappa_{1}\left(\xi_{1}\right) \xi_{2} \\
& \dot{\xi}_{2}=\kappa_{2}\left(\xi_{1}\right),
\end{aligned}
$$

where $\xi_{1}=\theta(q), \xi_{2}=d_{N}(q) \dot{q}$, [1]. By Property $1, \xi_{2}$ is the angular momentum about the stance foot, $\sigma$.

Definition 1: Let $Z$ and $\dot{z}=f_{\text {zero }}(z)$ be the zero dynamics manifold of the single support phase and the associated zero dynamics, respectively. $Z$ is a hybrid zero dynamics manifold if $\forall z^{-} \in S \cap Z, \exists F_{f} \in \mathbb{R}^{+} \cup\{0\}$ such that $\Delta\left(z^{-}, F_{f}\right) \in Z$. The nonlinear system

$$
\begin{array}{ll}
\dot{z}=f_{\text {zero }}(z), & z^{-} \notin S \cap Z \\
z^{+}=\Delta\left(z^{-}, F_{f}\right), & z^{-} \in S \cap Z, F_{f} \in \mathbb{R}^{+} \cup\{0\}
\end{array}
$$

with $z \in Z$ is called the hybrid zero dynamics of (10).

Remark 1: By the definition, $Z$ is a hybrid zero dynamic manifold if, and only if, $\forall z^{-} \in S \cap Z, \exists F_{f} \in \mathbb{R}^{+} \cup\{0\}$ such that

$$
\begin{aligned}
h \circ \Delta\left(z^{-}, F_{f}\right) & =0, \\
L_{f} h \circ \Delta\left(z^{-}, F_{f}\right) & =0 .
\end{aligned}
$$

Let $x(t)$ be a solution of the system (10) for the single support phase with the output $y=h(x)$ being zero. If the trajectory of the solution contains an impact with $S$ then the impact time $t_{I}$ exists and is finite. Then $x^{-}:=\lim _{t / t_{I}} x(t)$ exists and $x^{-}=\left(q^{-}, \dot{q}^{-}\right)^{\prime} \in S \cap Z$. By HH5, $q^{-}$is a unique point in $\tilde{\mathcal{Q}}$ defined as in HH2. Define $\Phi: \mathbb{R} \rightarrow S \cap Z$ as

$$
\Phi=\left[\begin{array}{c}
\Phi_{q} \\
\Phi_{\dot{q}}
\end{array}\right],
$$

where $\Phi_{q}=q^{-}$and

$$
\Phi_{\dot{q}}\left(q^{-}\right)=\left[\begin{array}{c}
\frac{\partial h}{\partial q}\left(q^{-}\right) \\
d_{N}\left(q^{-}\right)
\end{array}\right]^{-1}\left[\begin{array}{l}
0 \\
1
\end{array}\right],
$$

then $\Phi$ is a diffeomorphism, [1]. Define $z^{-}=\left(\theta^{-}, \sigma^{-}\right)=$ $\left(\theta\left(q^{-}\right), d_{N}\left(q^{-}\right) \dot{q}^{-}\right)$then the double support map for the velocity states $(8)$ becomes

$$
\begin{aligned}
\dot{q}^{+} & =\Delta_{\dot{q} 1}\left(q^{-}\right) F_{f}+\Delta_{\dot{q} 2}\left(q^{-}\right) \Phi_{\dot{q}}\left(q^{-}\right) \sigma^{-} \\
& =w_{1} F_{f}+w_{2} \sigma^{-},
\end{aligned}
$$

where $w_{1}=\Delta_{\dot{q} 1}\left(q^{-}\right)$and $w_{2}=\Delta_{\dot{q} 2}\left(q^{-}\right) \Phi_{\dot{q}}\left(q^{-}\right)$. Note that $w_{1}, w_{2}$ are uniquely defined $N \times 1$ vectors.

Let $Z$ be the hybrid zero dynamics manifold. The double support map for $\theta$ restricted to $Z$ is given by

$$
\theta^{+}=\theta \circ \Delta_{q}\left(q^{-}\right) .
$$

The double support map of $\sigma$ restricted to $Z$ is given by

$$
\begin{aligned}
\sigma^{+} & =d_{N}\left(q^{+}\right) \dot{q}^{+} \\
& =d_{N}\left(q^{+}\right)\left(\Delta_{\dot{q} 2}\left(q^{-}\right) \Phi_{\dot{q}} \sigma^{-}+\Delta_{\dot{q} 1}\left(q^{-}\right) F_{f}\right) .
\end{aligned}
$$

Using Properties 1 to 6 , it follows that (20) is equivalent to

$$
\sigma^{+}=\sigma^{-}+l\left(M v_{c}^{v-}+\cos \phi_{1} F_{f}\right),
$$

where $l$ is the step length, $M$ is the total mass, and $v_{c}^{v-}$ is the vertical component of the velocity of the center of mass just before the impact.

Theorem 1: Suppose there exists at least one point $z^{-} \in$ $S \cap Z$ and $F_{f}$ such that $\sigma^{-} \neq 0, h \circ \Delta\left(z^{-}, F_{f}\right)=$ 0 , and $L_{f} h \circ \Delta\left(z^{-}, F_{f}\right)=0$. Then $\forall z^{-} \in S \cap Z$, $L_{f} h \circ \Delta\left(z^{-}, F_{f}\right)=0$ if, and only if, one of the following is satisfied.

1) $F_{f}=r_{f} \sigma^{-}$for $r_{f}$ a constant;

2) $\frac{\partial h}{\partial q}\left(\Delta_{q}\left(q^{-}\right)\right) w_{1}=0$ and $\frac{\partial h}{\partial q}\left(\Delta_{q}\left(q^{-}\right)\right) w_{2}=0$, where $w_{1}, w_{2}$ are the vectors defined in (18).

Proof: Proof follows from the fact the null space of $\frac{\partial h}{\partial q}\left(\Delta_{q}\left(q^{-}\right)\right)$is one dimensional and the vector $w_{1} F_{f}+$ $w_{2} \sigma^{-}$is in the null space.

Although having an output function satisfying the second condition in Theorem 1 enables us to use an arbitrary foot force to achieve the hybrid zero dynamics, designing such an output function is too restrictive. So the foot force during the double support phase is chosen as

$$
F_{f}=r_{f} \sigma^{-}
$$

to create the hybrid zero dynamics. Then the double support map (20) becomes

$$
\begin{aligned}
\sigma^{+} & =d_{N}\left(q^{+}\right)\left(\Delta_{\dot{q} 1} r_{f}+\Delta_{\dot{q} 2}\right) \sigma^{-} \\
& =\delta_{z} \sigma^{-},
\end{aligned}
$$

where $\delta_{z}=d_{N}\left(q^{+}\right)\left(\Delta_{\dot{q} 1} r_{f}+\Delta_{\dot{q} 2}\right)$. In general, designing the output function for $k$ th step to achieve the hybrid zero dynamics requires knowledge of the foot force applied during the prior double support phase. In this paper, the foot force is chosen in such a way that we can design the output function a priori so that it is identical for each step.

\section{STABILITY ANALYSIS}

Using Poincaré map, the stability of the robot restricted to the hybrid zero dynamics manifold is analyzed. Then the stability of the full model is addressed.

In [1], it was shown that if the robot completes a step with the zero dynamics, $\sigma$ is not zero during the single support phase. Since $\sigma \neq 0$ during the step, $\zeta=\frac{\sigma^{2}}{2}$ is a valid coordinate transformation. Then, (11) and (12) become

$$
d \zeta=\sigma d \sigma=\frac{\kappa_{2}(\theta)}{\kappa_{1}(\theta)} d \theta .
$$

Let $z^{-}=\left(\theta^{-}, \sigma^{-}\right) \in S \cap Z$ and $\theta^{+}$be defined as in (19). For $\theta^{+} \leq \theta \leq \theta^{-}$, define

$$
\begin{aligned}
V_{\text {zero }}(\theta) & =-\int_{\theta^{+}}^{\theta} \frac{\kappa_{2}(\xi)}{\kappa_{1}(\xi)} d \xi, \\
V_{\text {zero }}^{\max } & =\max _{\theta^{+} \leq \theta \leq \theta^{-}} V_{\text {zero }}(\theta) .
\end{aligned}
$$

If $\delta_{z}^{2} \zeta^{-}-V_{z e r o}^{\max }>0$, then (24) can be integrated over a step, which results in

$$
\frac{1}{2}\left(\sigma^{-}\right)^{2}-\frac{1}{2}\left(\sigma^{+}\right)^{2}=\zeta^{-}-\zeta^{+}=-V_{\text {zero }}\left(\theta^{-}\right) .
$$


From (23), $\zeta^{+}=\delta_{z}^{2} \zeta^{-}$. Hence, the reduced Poincaré map in $(\theta, \zeta)$ coordinates, $\rho\left(\zeta^{-}\right): S \cap Z \rightarrow S \cap Z$, is defined as follows

$$
\rho\left(\zeta^{-}\right)=\delta_{z}^{2} \zeta^{-}-V_{z e r o}\left(\theta^{-}\right)
$$

with domain of definition

$$
\mathcal{D}=\left\{\zeta^{-}>0 \mid \delta_{z}^{2} \zeta^{-}-V_{\text {zero }}^{\max }>0\right\} .
$$

Theorem 2: Under the hypotheses RH1-RH5, GH1GH5, IH1-IH6, and HH1-HH5 in Appendix A and IFH1IFH7,

$$
\zeta^{*}=-\frac{V_{\text {zero }}\left(\theta^{-}\right)}{1-\delta_{z}^{2}}
$$

is an exponentially stable fixed point of (28) if, and only if,

$$
\begin{aligned}
& 0<\delta_{z}^{2}<1 \\
& \frac{\delta_{z}^{2}}{1-\delta_{z}^{2}} V_{\text {zero }}\left(\theta^{-}\right)+V_{z e r o}^{\max }<0 .
\end{aligned}
$$

Proof: $\mathcal{D}$ is non-empty if, and only if, $\delta_{z}^{2}>0$. If there exists $\zeta^{*} \in \mathcal{D}$ satisfying $\rho\left(\zeta^{*}\right)=\delta_{z}^{2} \zeta^{*}-V_{z e r o}$, then $\zeta^{*}$ is an exponentially stable fixed point if, and only if, $0<\delta_{z}^{2}<1$, and in this case, (30) is the value of $\zeta^{*}$. Finally, (32) is the necessary and sufficient condition for (30) to be in $\mathcal{D}$.

Note that this is identical to the result of [1] if $r_{f}$ is set to be zero. Using (21), the condition (31) can be interpreted as $M v_{c}^{v-}$, the value of the vertical component of the robot's linear momentum, should be negative just before impact, and for stability, $\cos \phi_{1} F_{f}$, the intensity of the vertical component of the foot force, should not exceed $\left|M v_{c}^{v-}\right|$.

Differentiating the output $y=h(x)$ twice gives

$$
\begin{aligned}
\ddot{y} & :=v \\
& =L_{f}^{2} h(x)+L_{g} L_{f} h(x) u .
\end{aligned}
$$

Let $v$ be any control input satisfying controller hypotheses CH2-CH5 in [1], (see Appendix A). Then the zero dynamics manifold for the single support phase is invariant. In this paper, the controller by Bhat and Bernstein, [18] is used, see Appendix D. The feedback for the full-model (10) is given as follows,

$$
u=\left(L_{g} L_{f} h(x)\right)^{-1}\left(v\left(h(x), L_{f} h(x)\right)-L_{f}^{2} h(x)\right) .
$$

By Theorem 2 in [5], the full state model is asymptotically stable if and only if the reduced Poincaré map (28) has an exponentially stable fixed point. Therefore, the full system is asymptotically stable.

\section{PARAMETERIZATION AND OPTIMIZATION OF THE HYBRID ZERO DYNAMICS}

In this section, output functions and foot actuation are designed so as to create a hybrid zero dynamics for the system (10). As in [1], the fundamental idea is to design a zero dynamics of the single support phase model that is also invariant under the double support map. Controlling the robot is realized by imposing constraints as an output function, which is driven to zero using feedback. Therefore the feedback is determined by the choice of the output function $h(q)$. Thus, designing an optimal feedback $u$ is equivalent to designing an optimal output $h(q)$.

The output $y=h(q)$ is defined as

$$
y=h(q)=H_{0} q-h^{d}(\theta(q)),
$$

where $H_{0}$ is a $(N-1) \times N$ constant matrix and $h^{d}(\theta(q))$ is a desired function for $H_{0} q$ to track and $\theta(q)=c q$ satisfies HH3 so that $\left[\begin{array}{ll}H_{0}^{\prime} & c^{\prime}\end{array}\right]^{\prime}$ has full rank. The desired function $h^{d}(\theta(q))=h^{d}(s) \circ \theta(q)$ is a $(N-1) \times 1$ vector whose elements are Bézier polynomials. Let $b_{i}(s)$ be Bézier polynomial with order $M \geq 3$ as

$$
b_{i}(s):=\sum_{k=0}^{M} \alpha_{k}^{i} \frac{M !}{k !(M-k) !} s^{k}(1-s)^{M-k},
$$

then $h^{d}(\theta(q))=\left[b_{i}(s), \cdots, b_{N-1}(s)\right]^{\prime}$ and

$$
s(\theta(q))=\frac{\theta(q)-\theta^{+}}{\theta^{-}-\theta^{+}} .
$$

Let $\alpha_{k}=\left[\alpha_{k}^{1}, \cdots, \alpha_{k}^{N-1}\right]^{\prime}$ where the constants $\alpha_{k}^{i}$ are the coefficients of the Bézier polynomials in (37). Let the foot force be defined as in (22). Then (14) and (15) are satisfied when

$$
\left[\begin{array}{l}
\alpha_{0} \\
\theta^{+}
\end{array}\right]=H R H^{-1}\left[\begin{array}{c}
\alpha_{M} \\
\theta^{-}
\end{array}\right]
$$

and

$$
\alpha_{1}=\frac{H_{0}\left(\theta^{-}-\theta^{+}\right)\left(\Delta_{\dot{q} 2} \Phi_{\dot{q}}\left(q^{-}\right)+\Delta_{\dot{q} 1} r_{f}\right)}{M c\left(\Delta_{\dot{q} 2} \Phi_{\dot{q}}\left(q^{-}\right)+\Delta_{\dot{q} 1} r_{f}\right)}+\alpha_{0} .
$$

Note that since the foot actuation does not affect the position states, the condition for $\alpha_{0}$ to satisfy (14) is identical to the model of unactuated point feet. The condition for $\alpha_{1}$ to satisfy (15) implies that, with $F_{f}$ defined as in (22), the output function $y=h(q)$ can be determined a priori. An optimal control can be found by optimizing the output parameters. The cost function to be optimized is defined as

$$
J=\frac{1}{L_{s}}\left(W_{j}+W_{f}\right),
$$

where $L_{s}$ is the step length, $W_{j}$ is the sum of the work done by the joints, and $W_{f}$ is the work done by foot actuation.

The work done by each joint is defined as

$$
W_{j}=\sum_{i=1}^{N-1} \int_{T_{s}^{+}}^{T_{s}^{-}} \dot{q}_{i} u_{i} d t,
$$

where $T_{s}^{+}, T_{s}^{-}$represent the time of beginning and end of a step.

The work done by the impulsive foot action is defined as follows. Let $t_{I}$ be the impact time and $\tau=E_{e}^{1} \delta F_{1}=$ $E_{e}^{1} F_{1} \delta\left(t-t_{I}\right)$ from (2) be the effective impulsive torque of the foot. Then the work by the impulsive foot actuation is given by

$$
W_{f}=\int_{t_{I}^{-}}^{t_{I}^{+}} \tau^{T} \dot{q}_{e} d t=\int_{t_{I}^{-}}^{t_{I}^{+}}\left(E_{e}^{1} F_{1} \delta\left(t-t_{I}\right)\right)^{T} \dot{q}_{e} d t .
$$


Due to the impulsive foot actuation, there exists a discontinuous change in the velocity states. Let $\Delta \dot{q}_{e}=\dot{q}_{e}^{+}-\dot{q}_{e}^{-}$ then the velocity states can be written as

$$
\dot{q}_{e}=\Delta \dot{q}_{e} u\left(t-t_{I}\right)+\dot{\bar{q}}_{e},
$$

where $u\left(t-t_{I}\right)$ is a step function. Note that $\dot{\bar{q}}_{e}$ is continuous and $\Delta \dot{q}_{e}$ can be obtained from (3) as

$$
\Delta \dot{q}_{e}=\left(D_{e}\right)^{-1} E_{e}^{1} F_{1} .
$$

From (43) and (44), the work done by foot actuation can be obtained as

$$
\begin{aligned}
W_{f}= & \left(E_{e}^{1} F_{1}\right)^{T} \Delta \dot{q}_{e} \int_{t_{I}^{-}}^{t_{I}^{+}} \delta\left(t-t_{I}\right) u\left(t-t_{I}\right) d t \\
& +\left(E_{e}^{1} F_{1}\right)^{T} \dot{q}_{e}^{-} .
\end{aligned}
$$

By Hypothesis GH2 in Appendix A, which is the stance leg acting as a pivot during the single support phase,

$$
\frac{\partial \Upsilon_{1}\left(q_{e}\right)}{\partial q_{e}} \dot{q}_{e}^{-}=\left(E_{e}^{1}\right)^{T} \dot{q}_{e}^{-}=0,
$$

where $\Upsilon_{1}\left(q_{e}\right)$ is the Cartesian coordinates of the stance leg. With (47) and (45), the work by the foot becomes

$$
W_{f}=\left(E_{e}^{1} F_{1}\right)^{T}\left(D_{e}\right)^{-1} E_{e}^{1} F_{1} \int_{t_{I}^{-}}^{t_{I}^{+}} \delta\left(t-t_{I}\right) u\left(t-t_{I}\right) d t .
$$

For the work by the foot to be calculated, it is required to determine the value of $\int_{t_{I}^{-}}^{t_{I}^{+}} \delta\left(t-t_{I}\right) u\left(t-t_{I}\right) d t$. Although the integration is not defined at $t=t_{I}$ in general, in this paper it is taken to be $\int_{t_{I}^{-}}^{t_{I}^{+}} \delta\left(t-t_{I}\right) u\left(t-t_{I}\right) d t=\frac{1}{2}$.

Since the hybrid zero dynamics needs to be achieved during walking, (39) and (40) should be satisfied. Therefore, only $(N-1) \times(M-1)$ coefficients of (37) need to be determined. The detailed constraints for optimization are given in [1], which ensure a valid stable walking motion.

IMPULSIVE FOOT CONSTRAINT: An additional constraint on foot actuation is necessary to ensure the applied foot force is unilateral.

IFC1) Foot force, $\delta F_{f}$, is unilateral.

\section{EXAMPLE}

This section illustrates the above results on a robot with impulsive foot action. The robot is a 5-link planar bipedal robot [19] with an impulsive actuator at each foot. For the output function, the order of the Bézier polynomials is set to be $M=6$. The constraint on the average walking speed is $0.95(\mathrm{~m} / \mathrm{s})$. For optimization, the fmincon function in MATLAB, which utilizes SQP, was used. Table I shows the work done by the joint actuators and the foot actuator. The work is normalized by the distance traveled during one step (41). The normalized work done by the robot with foot actuation is less than the robot without foot actuation, which implies that having foot actuation helps to reduce the work required to walk.

\begin{tabular}{|c|c|c|c|c|}
\hline$F_{f}(N)$ & $L_{s}(\mathrm{~m})$ & $W_{\text {total }}(J / m)$ & $W_{j}(J / m)$ & $W_{f}(J / m)$ \\
\hline \hline 0 & 0.45 & 38.32 & 38.32 & 0 \\
\hline 11.53 & 0.58 & 28.79 & 24.74 & 4.05 \\
\hline
\end{tabular}

TABLE I

WORK DONE BY THE JOINTS AND THE FOOT PER DISTANCE TRAVELED. $F_{f}, L_{s}$ REPRESENT THE FOOT FORCE AND STEP LENGTH, $W_{\text {total }}, W_{j}$, AND $W_{f}$ REPRESENT TOTAL WORK, WORK BY JOINTS, AND WORK BY FOOT PER DISTANCE TRAVELED, RESPECTIVELY.

Fig. 3 and Fig. 4 show the applied torque at each joint. $u_{1}, u_{2}, u_{3}$, and $u_{4}$ represent the torques applied at the stance hip, the swing hip, the stance knee, and the swing knee, respectively. Fig. 3 is the plot of torques of the robot without foot actuation and Fig. 4 is the plot of torques of the robot with foot actuation. The robot with foot actuation requires smaller torques during walking. In particular, smaller torques between the torso and two femurs of the robot with foot actuation are applied.
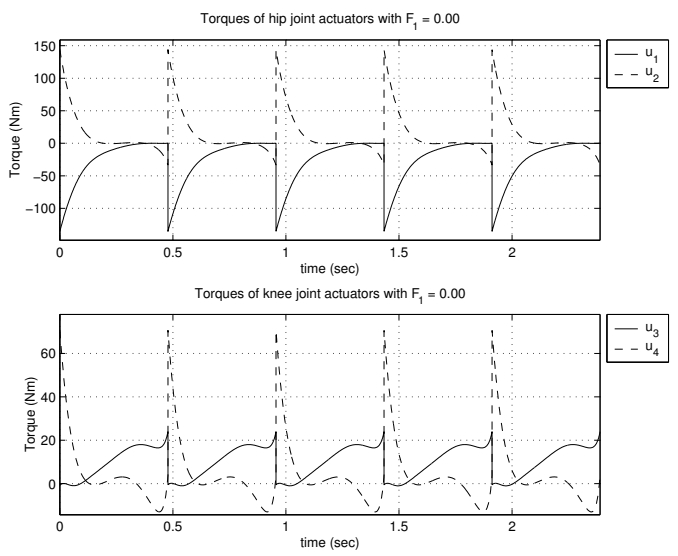

Fig. 3. Torques of the robot without foot actuation. Dashed lines are torques of the swing leg joints and solid lines are torques of the stance leg joints.
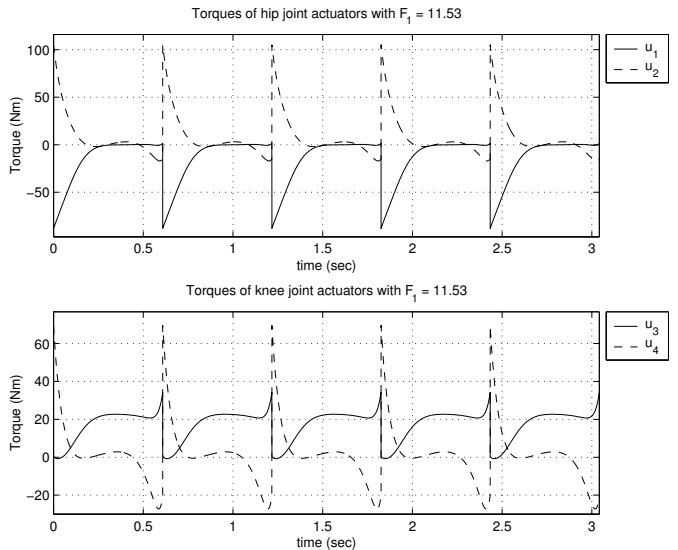

Fig. 4. Torques of the robot with foot actuation. Dashed lines are torques of the swing leg joints and solid lines are torques of the stance leg joints. 


\section{CONCLUSION}

A robot model with impulsive foot actuation was introduced. The model was developed from a model with unactuated point feet [1] and the work in [2]. The impulsive actuator at the end of the stance foot was assumed to be active only in the double support phase, which resulted in the identical model to the robot with unactuated point feet for the single support phase. However, the double support map depends on the foot force, which results in changes in the zero dynamics. In general, the output function cannot be designed to achieve the hybrid zero dynamics without knowing the foot force a priori. A method to design output functions to achieve the hybrid zero dynamics was given for a class of foot actuation control policies. The stability of the resulting walking was shown with a Poincaré map.

\section{APPENDIX}

\section{A. Hypotheses}

The following hypotheses are based on the hypotheses in [1]. The hypotheses for the robot are:

$\mathrm{RH} 1)$ The robot consists of $\mathrm{N}$ rigid links with revolue joint;

RH2) The robot is planar;

RH3) The robot is bipedal with identical legs connected at hips;

RH4) The joints are actuated;

RH5) The coordinate of the robot consists of $N-1$ relative angles, $q_{1}, \cdots, q_{N-1}$, and one absolute angle, $q_{N}$

The hypotheses for gait are:

GH1) Walking consists of two alternating phases, single support phase and double support phase;

GH2) The stance feet acts as a pivot during the single support phase;

GH3) The swing leg has neither slipping nor rebounding at impact;

GH4) Successive single support phases are identical with respect to the two legs in steady state;

GH5) Walking is from left to right, so that the swing leg moves from behind the stance leg and touches the ground in front of the stance leg at impact.

The hypotheses for impact are:

IH1) The swing leg has neither rebound nor slipping during impact;

IH2) After impact, the stance leg leaves the ground without any interaction with the ground;

IH3) The impact is instantaneous;

IH4) The reaction force due to the impact can be modeled as an impulse;

IH5) The impulsive force results in discontinous changes in the velocities while the position states remain continuous;

IH6) The actuators at joints are not impulsive.

Let the output $y$ be $y=h(x)$ then the hypotheses are:

HH1) The output function $h(x)$ being a function of only the configuration coordinates during the single support phase;

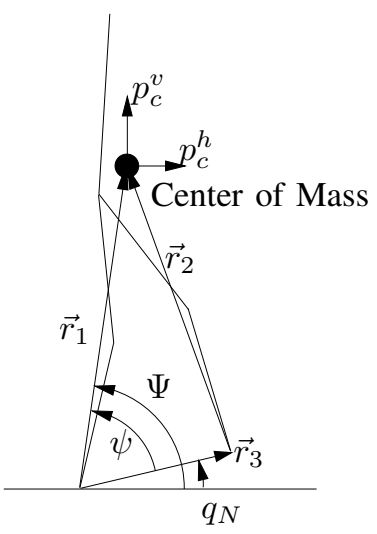

Fig. 5. Coordinates of the center of mass

HH2) The decoupling matrix $L_{g} L_{f} h$ being invertible for an open set $\tilde{\mathcal{Q}} \subset \mathcal{Q}$;

HH3) Existence of $\theta(q)$ such that $[h(q) ; \theta(q)]$ is diffeomorphism;

HH4) $h$ vanishing at least one point.

HH5) Existence of an unique point $q^{-} \in \tilde{\mathcal{Q}}$ such that $\left(h\left(q^{-}\right), p_{2}^{v}\left(q^{-}\right)\right)=(0,0), p_{2}^{h}\left(q^{-}\right)>0$ and the rank of $\left[h^{\prime} p_{2}^{v}\right]^{\prime}$ at $q^{-}$equals to $N$.

The hypotheses for the closed-loop chain of double integrators, $\ddot{y}=v$, are:

CH1) Global invertibility of the decoupling matrix.

$\mathrm{CH} 2$ ) Existence of solutions on $\mathbb{R}^{2 N-2}$ and uniqueness;

$\mathrm{CH} 3)$ Solutions depending continuously on the initial conditions;

$\mathrm{CH} 4)$ The origin being globally asymptotically stable and the convergence being achieved in finite time;

CH5) The settling time depending continuously on the initial condition.

\section{B. Proof of property 1}

Proof: Let $\left(p_{c}^{h}, p_{c}^{v}\right)$ be the Cartesian coordinates of the center of mass of the robot. Let $\vec{r}_{1}$ be a vector from the stance foot to the center of mass and let $\vec{r}_{2}$ be the vector from the stance foot to the swing foot. Let $\Psi$ and $\psi$ be the angle between $\vec{r}_{1}$ and the ground, and the angle between $\vec{r}_{1}$ and $\vec{r}_{2}$, respectively. Let $q_{N}$ be the absolute angle of $\vec{r}_{3}$ calculated from the ground, see Fig. 5.

Then $\left|\vec{r}_{1}\right|$ and $\psi$ are independent from $q_{N}$ and $\Psi=\psi+$ $q_{N}$. Therefore, the coordinate of the center of mass is

$$
\begin{aligned}
& p_{c}^{h}=\left|\vec{r}_{1}\right| \cos \left(\psi+q_{N}\right) \\
& p_{c}^{v}=\left|\vec{r}_{1}\right| \sin \left(\psi+q_{N}\right) .
\end{aligned}
$$

The partial derivatives of (49) and (50) with respect to $q_{N}$ are given as

$$
\begin{aligned}
& \frac{\partial p_{c}^{h}}{\partial q_{N}}=-\left|\vec{r}_{1}\right| \sin \left(\psi+q_{N}\right)=-p_{c}^{v} \\
& \frac{\partial p_{c}^{v}}{\partial q_{N}}=\left|\vec{r}_{1}\right| \cos \left(\psi+q_{N}\right)=p_{c}^{h} .
\end{aligned}
$$


The Lagrangian is defined as $L=K-V$, where $K$ is the kinetic energy and $V$ is the potential energy of the robot. Since $K$ does not depend on $q_{N}$ and $V=M g p_{c}^{v}$ with $g$ being gravitational acceleration and $M$ being total mass,

$$
\frac{d}{d t} \frac{\partial L}{\partial \dot{q}_{N}}=\frac{\partial L}{\partial q_{N}}=-\frac{\partial V}{\partial q_{N}}=-M g p_{c}^{h} .
$$

Let $\sigma$ be the angular momentum about the stance foot. Then,

$$
\frac{d \sigma}{d t}=\left(\vec{r}_{1} \times \vec{F}_{c}\right) \cdot \vec{e}_{3}
$$

where $\vec{F}_{c}$ is the force acting on the center of mass. Since $\vec{r}_{1}=p_{c}^{h} \vec{e}_{1}+p_{c}^{v} \vec{e}_{2}$ and $\vec{F}_{c}=-M g \vec{e}_{2}$,

$$
\frac{d \sigma}{d t}=-M g p_{c}^{h}
$$

From (53) and (55), we can deduce that $\partial L / \partial \dot{q}_{N}=\sigma+C$, with some constant $C$. Since $\partial L / \partial \dot{q}_{N}$ and $\sigma$ are zero if $\left(\dot{q}_{1}, \cdots, \dot{q}_{N}\right)=0, C=0$. Therefore

$$
\frac{\partial L}{\partial \dot{q}_{N}}=\sigma .
$$

Since $d_{N}(q) \dot{q}=\partial L / \partial \dot{q}_{N}, d_{N}(q) \dot{q}$ is the angular momentum of the robot.

\section{Proof of property 4}

Proof: Let $q_{c}=\left(q_{1}, \cdots, q_{N}, p_{c}^{h}, p_{c}^{v}\right)$ be the coordinates of the robot, where $p_{c}^{h}, p_{c}^{v}$ denote the Cartesian coordinates of the center of mass, see Fig. 5. Then the kinetic energy $K_{e}$ does not depend on $p_{c}^{h}, p_{c}^{v}$ and the potential energy is given by $V_{e}=M g p_{c}^{v}$. Since the force acting on the center of mass is $F_{h}=0, F_{v}=-M g$,

$$
\begin{aligned}
\frac{d}{d t} \frac{\partial L_{e}}{\partial \dot{p}_{c}^{h}} & =0=F_{h} \\
\frac{d}{d t} \frac{\partial L_{e}}{\partial \dot{p}_{c}^{v}} & =-M g=F_{v} .
\end{aligned}
$$

Therefore, $\frac{\partial L_{e}}{\partial \dot{p}_{c}^{h}}, \frac{\partial L_{e}}{\partial \dot{p}_{c}^{v}}$ are the linear momentum. In the coordinates $q_{c}$, let $\Upsilon_{1}\left(q_{c}\right)$ be the Cartesian coordinates of the stance foot. Then, $\vec{P}_{c}^{+}-\vec{P}_{c}^{-}=\mathcal{T} F_{f}$.

\section{Finite settling time controller for double integrators}

The following controller is the finite-settling time controller for a double integrator developed by Bhat and Bernstein, [18]. Consider the double integrator

$$
\begin{aligned}
& \dot{x}=y \\
& \dot{y}=u .
\end{aligned}
$$

The double integrator (59) is globally finite-time stabilizable with continuous feedback control

$$
u=-\operatorname{sign}(y)|y|^{\alpha}-\operatorname{sign}\left(\phi_{\alpha}(x, y)\right)\left|\phi_{\alpha}(x, y)\right|^{\frac{\alpha}{2-\alpha}},
$$

where $\phi_{\alpha}(x, y):=x+\frac{1}{2-\alpha} \operatorname{sign}(y)|y|^{2-\alpha}$ and $0<\alpha<1$. The settling time, $T_{\text {set }}$, depends continuously on the initial condition.

\section{ACKNOWLEDGMENTS}

This work was supported by NSF grant ECS-0322395.

\section{REFERENCES}

[1] E. R. Westervelt, J. W. Grizzle, and D. E. Koditschek, "Hybrid zero dynamics of planar biped walkers," IEEE Trans. Automatic Control, vol. 48, no. 1, pp. 42-56, 2003.

[2] A. D. Kuo, "Enegetics of actively powered locomotion using the simplest walking model," J. of Biomechanical Engineering, vol. 124, pp. 113-120, February 2002.

[3] G. A. Cavagna and R. Margaria, "Mechanics of walking," J. of Applied Physiology, vol. 21, no. 1, pp. 271-278, 1966.

[4] A. Goswami, "Postural stability of biped robot and the foot-rotation indicator (FRI) point," Int. J. of Robotics Research, vol. 18, no. 6, pp. 523-533, June 1999.

[5] J. W. Grizzle, G. Abba, and F. Plestan, "Asymptotically stable walking for biped robots: Analysis via systems with impulse effects," IEEE Trans. Automatic Control, vol. 46, no. 1, pp. 51-64, 2001.

[6] T. McGeer, "Passive dynamic walking," Int. J. of Robotics Research, vol. 9, no. 2, pp. 62-82, 1990.

[7] E. R. Westervelt, G. Buche, and J. W. Grizzle, "Inducing dynamically stable walking in an underactuated prototype planar biped," in IEEE 2004 Int. Conf. on Robotics and Automation, October 2003, to appear; see http://www.mecheng.ohio-state.edu/ ${ }^{\sim}$ westerve for a preprint.

[8] E. Westervelt, G. Buche, and J. Grizzle, "Experimental validation of a framework for the design of controllers that induce stable walking in planar bipeds," The Int. J. of Robotics Research, to appear; see http://www.mecheng.ohio-state.edu/ ${ }^{\sim}$ westerve for a pre-print.

[9] K. Y. Yi, "Walking of a biped robot with passive ankle joints," in Proc. Int. Conf. Control Application, Kohala Coast-Island of Hawaii, Hawaii, USA, August 1999, pp. 484-489.

[10] R. Q. van der Linde, "Active leg compliance for passive walking," in Proc. Int. Conf. Robotics and Automation, Leuven, Belgium, May 1998, pp. 2339-2344.

[11] K. Hirai, M. Hirose, Y. Haikawa, and T. Takenaka, "The development of honda humanoid robot," in Proc. Int. Conf. Robotics and Automation, Leuven, Belgium, May 1998, pp. 1321-1326.

[12] H.-O. Lim, S. A. Setiawan, and A. Takanishi, "Balance and impedance control for biped humanoid robot locomotion," in Proc. IEEE/RSJ Int. Conf. Intelligent Robots and Systems, Maui, Hawaii, October 2001, pp. 494-499.

[13] C.-L. Shih, "The dynamics and control of a biped walking robot with seven degrees of freedom," J. of Dynamic Systems, Measurement, and Control, vol. 118, pp. 683-690, December 1996.

[14] A. Sano and J. Furusho, "Realization of natural dynamic walking using the angular momentum information," in Proc. IEEE Int. Conf. Robotica and Automation, Cincinnati, OH, May 1990, pp. 14761481.

[15] T. Takahashi and A. Kawamura, "Posture control using foot toe and sole for biped walking robot "ken"," in Int. Workshop on Advanced Motion Control, Maribor, Slovenia, 2002, pp. 437-442.

[16] [Online]. Available: http://tam.cornell.edu//ruina/hplab/pdw.html

[17] H. Ye, A. N. Michel, and L. Hou, "Stability theory for hybrid dynamical systems," IEEE Trans. Automatic Control, vol. 43, no. 4, pp. 461-474, April 1998.

[18] S. P. Bhat and D. S. Bernstein, "Continuous finite-time stabilization of the translational and rotational double integrators," IEEE Trans. Automatic Control, vol. 43, no. 5, pp. 678-682, May 1998.

[19] C. Chevallereau, G. Abba, Y. Aoustin, F. P. andE. R. Westervelt, C. C. de Wit, and J. W. Grizzle, "Rabbit: A testbed for advanced control theory," IEEE Control Systems Magazine, vol. 23, no. 5, pp. 57-79, October 2003. 